\title{
MAGNETIC PROPERTIES OF NANOCRYSTALLINE Co/Ti AND Co/Zr MULTILAYERS
}

\author{
L. SMARDZ ${ }^{a}$ AND K. SMARDz ${ }^{b}$ \\ ${ }^{a}$ Institute of Molecular Physics, Polish Academy of Sciences \\ Smoluchowskiego 17, 60-179 Poznań, Poland \\ ${ }^{b}$ Institute of Mater. Sci. and Eng., University of Technology \\ Pl. Curie 5, 60-965 Poznań, Poland
}

\begin{abstract}
It is shown that the Co sublayers grow in the soft magnetic nanocrystalline phase up to a critical thickness $d \approx 3$ and $2.8 \mathrm{~nm}$ for $\mathrm{Co} / \mathrm{Ti}$ and $\mathrm{Co} / \mathrm{Zr}$ MLs, respectively. The rapid decrease in the interlayer exchange coupling could be explained by its strong damping due to formation of a non-magnetic quasi-amorphous $\mathrm{Co}-\mathrm{Ti}$ and $\mathrm{Co}-\mathrm{Zr}$ alloy layer at the interfaces. The nanocrystalline $2.2 \mathrm{~nm}-\mathrm{Co} / 2 \mathrm{~nm}-\mathrm{Ti} \mathrm{ML}$ shows a weak $90^{\circ}$ coupling near the transition zone from ferromagnetic to zero coupling.
\end{abstract}

PACS numbers: $75.70 .-\mathrm{i}, 75.30$.Et

Since the discovery of antiferromagnetic (AFM) interlayer coupling in $\mathrm{Fe} / \mathrm{Cr} / \mathrm{Fe}$ trilayers [1] magnetic exchange coupling of ferromagnetic sublayers across a crystalline spacer has been the subject of numerous experimental and theoretical studies. It has been shown that oscillatory exchange coupling is a general phenomenon for most transition-metal and noble-metal spacers [2]. The experimentally determined oscillation periods are in agreement with the theory based on the interplay between Ruderman-Kittel-Kasuya-Yoshida (RKKY) interaction and the discrete spacer thickness [3]. In contrast to the experimentally confirmed RKKY type mechanism of the oscillating bilinear exchange coupling, the origin of $90^{\circ}$-type coupling is not so clear [4]. Very recently Bürgler et al. [5] have shown that the amorphous metallic CuZr alloy spacer can mediate AFM exchange coupling between ferromagnetic layers. On the other hand, Fuchs et al. [6] found for the amorphous metallic AuSn alloy spacer ferromagnetic and $90^{\circ}$ coupling originating from dipolar interaction. In this paper we report on the magnetic properties (coercivity, anisotropy, and interlayer coupling) of the soft magnetic nanocrystalline $\mathrm{Co} / \mathrm{Ti}$ and $\mathrm{Co} / \mathrm{Zr}$ multilayers (MLs).

The $\mathrm{Co} / \mathrm{Ti}$ and $\mathrm{Co} / \mathrm{Zr}$ MLs were deposited onto $\mathrm{Si}(111)$ and glass using a UHV $\left(5 \times 10^{-10}\right.$ mbar $)$ magnetron sputtering. The Co-layers $\left(0.2 \leq d_{\text {Co }} \leq 20 \mathrm{~nm}\right)$ were deposited using a dc source. For preparation of $\mathrm{Ti}$ (or $\mathrm{Zr}$ ) layers 
$\left(0<d_{\mathrm{Ti}}\left(d_{\mathrm{Zr}}\right)<10 \mathrm{~nm}\right)$ an RF source was used. The chemical composition and cleanness of all layers was checked in situ, immediately after deposition, transferring the MLs to a UHV ( $4 \times 10^{-11}$ mbar) analyse chamber equipped with Auger and X-ray photoelectron spectroscopy (AES, XPS). From the exponential variation of the XPS Co- $2 p$, Ti-2p and $\mathrm{Zr}-3 p$ integral intensities with increasing layer thickness we conclude that the $\mathrm{Co}, \mathrm{Zr}$, and Ti sublayers grow homogeneously. The structure of the samples was examined $e x$ situ by standard $\theta-2 \theta$ X-ray diffraction. The modulation wavelength was determined from the spacing between satellite peaks in the low-angle $\mathrm{X}$-ray diffraction patterns. The results were consistent with values obtained from total thickness divided by the number of repetitions. The magnetic characterisation of the $\mathrm{Co} / \mathrm{Ti}$ and $\mathrm{Co} / \mathrm{Zr}$ MLs with constant-thickness sublayers was carried out using a magnetooptical Kerr effect at room temperature (RT) and a vibrating sample magnetometer in the temperature range 4-300 K, respectively. The coercive $\left(H_{\mathrm{c}}\right)$ and uniaxial anisotropy fields $\left(H_{k}\right)$ were determined from the in-plane hysteresis loops measurements at $295 \mathrm{~K}$.

Figure 1a shows $H_{\mathrm{c}}$ values, measured at room temperature, as a function of the Co sublayer thickness for the Co/Ti and Co/Zr MLs with $d_{\mathrm{Ti}}=2 \mathrm{~nm}$ and $d_{\mathrm{Zr}}=3 \mathrm{~nm}$. A significant drop of the coercivity - typically from $H_{\mathrm{c}} \approx 1.8(1.6) \mathrm{kA} / \mathrm{m}$ to $H_{\mathrm{c}} \approx 0.2 \mathrm{kA} / \mathrm{m}$ - can be observed for the $\mathrm{Co} / \mathrm{Ti}(\mathrm{Co} / \mathrm{Zr})$ MLs at a critical Co thickness $d \approx 3(2.8) \mathrm{nm}$. A possible explanation of the coercivity behaviour shown in Fig. 1a could be associated with the growth properties of the Co sublayer onto $\mathrm{Ti}$ or $\mathrm{Zr}$ sublayers. Cobalt sublayers grow in the soft magnetic nanocrystalline phase for a thickness lower than the critical one. In that case, the average Co grain size is significantly smaller than the magnetic exchange length for the cobalt layer $\left(L_{\mathrm{ex}} \approx 10 \mathrm{~nm}\right)$ [7]. For a thickness greater than $d$, the Co sublayers undergo a structural transition to the polycrystalline phase with average grains size $D>10 \mathrm{~nm}$ [7]. The structural transition from poly- to nanocrystalline phase was also characterised by a drastic broadening of the NMR spectra and decrease in the spin-spin relaxation time $[7,8]$. For the $\mathrm{Co} / \mathrm{Ti}$ and $\mathrm{Co} / \mathrm{Zr}$ MLs with polycrystalline Co sublayers we have observed small in-plane, highly dispersed uniaxial anisotropy $\left(H_{k} \approx 1 \mathrm{kA} / \mathrm{m}\right)$. The MLs with nanocrystalline Co sublayers showed well defined in-plane uniaxial anisotropy $\left(H_{k} \approx 2-5 \mathrm{kA} / \mathrm{m}\right)$.

The interlayer exchange coupling in the $\mathrm{Co} / \mathrm{Ti} / \mathrm{Co}$ and $\mathrm{Co} / \mathrm{Zr} / \mathrm{Co}$ trilayers was studied by measurements of the in-plane hysteresis loops as a function of the spacer thickness. The bottom and top Co sublayers of such structures were prepared in the different preparation conditions, so that the coercivities of the bottom sublayers were greater compared to those of the top sublayers $[7,9]$. The results showed that the Co sublayers are very weakly exchange coupled or decoupled for $\mathrm{Ti}$ and $\mathrm{Zr}$ spacer thickness equal to about 2 and $3 \mathrm{~nm}$, respectively. This was confirmed by the measurements of two significantly different coercivities (step-like hysteresis loops) in the trilayers. The small decoupling thicknesses could be explained by spontaneous formation of a quasi-amorphous structure of the paramagnetic spacer during the deposition process. In the case of the trilayers, the actual spacer consists of $\mathrm{Co}-\mathrm{Ti}(\mathrm{Co}-\mathrm{Zr})$ alloy and pure $\mathrm{Ti}(\mathrm{Zr})$ layer. As such alloy layers are non-magnetic at $295 \mathrm{~K}$ below the Co concentration of about 67 at.\% [7, 9], the spontaneously formed (during the deposition process) 

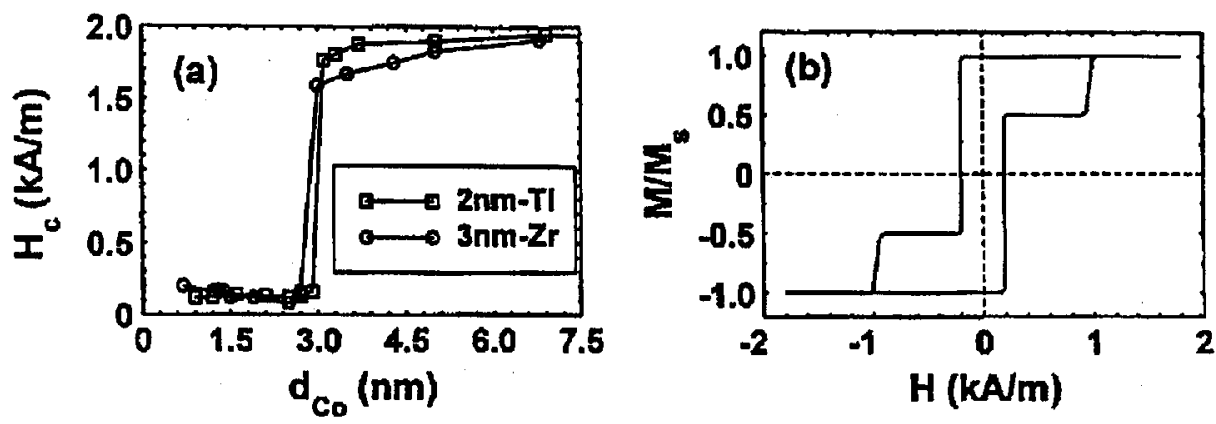

Fig. 1. Coercive field $\left(H_{c}\right)$ as a function of Co sublayer thickness for the Co/Ti and $\mathrm{Co} / \mathrm{Zr} \mathrm{MLs}$ (a). Hysteresis loop for the $2.2 \mathrm{~nm}-\mathrm{Co} / 2 \mathrm{~nm}-\mathrm{Ti} \mathrm{ML}$ with $90^{\circ}$ interlayer coupling (b).

alloy spacer at the $\mathrm{Co}-\mathrm{Ti}(\mathrm{Co}-\mathrm{Zr})$ interface acts as a paramagnetic spacer. On the other side, we have not observed any signal originating from the $\mathrm{Co}-\mathrm{Ti}$ interface alloy layer in the high-angle $\theta-2 \theta \mathrm{X}$-ray diffraction patterns. Therefore we conclude that the structure of this interface alloy layer is rather related to a quasi-amorphous (between nanocrystalline and amorphous) phase. Furthermore, it is well known that annealing of the Co/Ti and Co/Zr MLs leads to the formation of an amorphous phase due to solid state reaction [10]. Therefore the spontaneous formation of a quasi-amorphous spacer between the Co sublayers is very likely to proceed during the deposition process. This is consistent with the results of our X-ray diffraction and magnetisation studies of the Co/Ti MLs [7, 9]. The alloying effect at the $\mathrm{Co}-\mathrm{Ti}$ and $\mathrm{Co}-\mathrm{Zr}$ interfaces was also revealed very recently for the "as deposited" $\mathrm{Co} / \mathrm{Ti}$ and $\mathrm{Co} / \mathrm{Zr}$ MLs in the optical and magnetooptical studies $[11,12]$. In the case of such a quasi-amorphous structure of the spacer, a strong damping of the interlayer exchange coupling is to be expected by the recent theory based on the RKKY interaction [5]. The above effect could explain the estimated small decoupling thicknesses, observed for the $\mathrm{Co} / \mathrm{Ti}$ and $\mathrm{Co} / \mathrm{Zr}$ MLs. In addition, the amorphous $\mathrm{Co}-\mathrm{Ti}$ and $\mathrm{Co}-\mathrm{Zr}$ alloys approximately fulfil the Nagel-Tauc criterion $[5,7,9]$. Therefore for the amorphous $\mathrm{Co}-\mathrm{Ti}(\mathrm{Co}-\mathrm{Zr})$ alloy spacer, a large oscillation period of the exchange coupling is to be expected [5], so that the location of the first AFM maximum should appear for $d_{\mathrm{Ti}}>2 \mathrm{~nm}$ and $d_{\mathrm{Zr}}>3 \mathrm{~nm}$. On the other hand, due to the experimentally determined short exchange coupling range for the quasi-amorphous $\mathrm{Co}-\mathrm{Ti}(\mathrm{Co}-\mathrm{Zr})$ alloy spacer the first AFM maximum is practically not detectable [5, 9]. Therefore it is clear why we do not observe any AFM coupling for the Co/Ti and Co/Zr MLs. This is in agreement with results on interlayer coupling studies across amorphous AuSn alloy spacer [6].

In addition to the ferromagnetic interlayer coupling in the Co/Ti MLs with nanocrystalline Co sublayers $\left(d_{\mathrm{C}}<d\right)$, we have observed a weak $90^{\circ}$ coupling for a Ti thickness $d_{\mathrm{Ti}}=2 \mathrm{~nm}$, corresponding to the transition zone from ferromagnetic to zero exchange coupling. Figure $1 \mathrm{~b}$ shows the hysteresis loop of the $2.2 \mathrm{~nm}-\mathrm{Co} / 2 \mathrm{~nm}-\mathrm{Ti} \mathrm{ML}$. The steps at $M / M_{\mathrm{s}}=+0.5$ or -0.5 reveal the $90^{\circ}$ 
(negative biquadratic) coupling of the Co sublayers [5]. The saturation field $\left(H_{\mathrm{s}}\right)$ associated with the $90^{\circ}$ coupling is very low and after subtraction of the coercive field amounts about $0.8 \mathrm{kA} / \mathrm{m}$. The above value is significantly lower than the coercive fields of the polycrystalline Co/Ti MLs (see Fig. 1a). Therefore, the weak $90^{\circ}$ coupling could be only observed for the soft magnetic Co/Ti MLs with nanocrystalline Co sublayers. Our preliminary results on the temperature dependence of $H_{\mathrm{s}}$ rather exclude the "loose spins" mechanism [13] of such coupling. On the other hand, very short range of the observed $90^{\circ}$ coupling rather excludes the dipolar mechanism [6]. Furthermore, the assumption that the in-plane terrace size of the spacer is significantly greater than its thickness in the model with "fluctuating spacer thickness" mechanism [13], is no longer valid for the quasi-amorphous Co-Ti spacer. However, a similar physical mechanism based on the spacer thickness fluctuations may still be operative when the terrace width is comparable to the Ti sublayer thickness. The latter condition is satisfied in the case of the nanocrystalline $\mathrm{Co} / \mathrm{Ti}$ MLs [7].

In conclusion, the rapid decrease in the interlayer exchange coupling in the $\mathrm{Co} / \mathrm{Ti}(\mathrm{Co} / \mathrm{Zr})$ MLs could be explained by its strong damping due to formation of a non-magnetic quasi-amorphous $\mathrm{Co}-\mathrm{Ti}(\mathrm{Co}-\mathrm{Zr})$ alloy layer at the interfaces. The nanocrystalline $2.2 \mathrm{~nm}-\mathrm{Co} / 2 \mathrm{~nm}$-Ti ML shows a weak $90^{\circ}$ coupling near the transition zone from ferromagnetic to zero coupling.

\section{Acknowledgments}

This work was financially supported by the Committee for Scientific Research under grant No. 7 T08C 00914.

\section{References}

[1] P. Grünberg, R. Schreiber, M.B. Brodsky, H. Sower, Phys. Rev. Lett. 57, 2442 (1986).

[2] S.S.P. Parkin, Phys. Rev. Lett. 67, 3598 (1991).

[3] P. Bruno, C. Chappert, Phys. Rev. Lett. 67, 1602 (1991).

[4] P. Grünberg, M. Schäfer, K. Takanashi, U. Rücker, J. Nassar, I. Mertig, Acta Phys. Pol. A 91, 7 (1997).

[5] D.E. Bürgler, D.M. Schaller, C.M. Schmidt, F. Meisinger, J. Kroha, J. McCord, A. Hubert, H.J. Güntherodt, Phys. Rev. Lett. 80, 4983 (1998).

[6] P. Fuchs, U. Ramsperger, A. Vaterlaus, M. Landolt, Phys. Rev. B 55, 12546 (1997).

[7] K. Smardz, Ph.D. thesis, Institute of Molecular Physics, PAS, Poznań 1999.

[8] L. Smardz, K. Le Dang, H. Niedoba, K. Chrzumnicka, J. Magn. Magn. Mater. 140-144, 569 (1995).

[9] L. Smardz, K. Smardz, to be published.

[10] K. Samwer, Phys. Rep. 161, 3 (1988).

[11] G.M. Lee, K.W. Kim, Y.V. Kudryavtsev, L. Smardz, Y.P. Lee, Thin Solid Films 341, 165 (1999).

[12] Y.P. Lee, G.M. Lee, K.W. Kim, Y.V. Kudryavtsev, L. Smardz, J. Magn. Soc. Japan 23, 361 (1999).

[13] J.C. Slonczewski, J. Appl. Phys. 73, 5957 (1993). 